\title{
Radio Observations of Galactic SNRs
}

\author{
D.A. Green \\ Mullard Radio Astronomy Observatory, Cavendish Laboratory, \\ Madingley Road, Cambridge CB3 OHE, U.K.
}

\begin{abstract}
This paper reviews several aspects of the study of radio emission from Galactic Supernova Remnants (SNRs). The detection of radio emission from SNRs is the most important method for identification of SNRs, and the limitations imposed by selection effects on subsequent statistical studies are discussed. Also discussed are the spectral properties of SNRs, both their integrated spectra, and the evidence for spectral variations across the face of remnants.
\end{abstract}

\section{Introduction}

Observations at radio wavelengths are important for many studies of Galactic Supernova Remnants (SNRs), but it is not possible to review all of these in depth here. In particular, here I will not discuss the high-resolution radio images of Galactic SNRs, which reveal a lot of detail about the radio structure of SNRs and their interaction with the ISM, as these are largely based on high-frequency observations. Instead I will concentrate on reviewing the aspects of the radio emission from SNRs that are more dependent on wide-field, low-resolution or low-frequency data, namely a statistical overview of the radio properties of SNRs Galactic SNRs, and studies of their radio spectral properties.

\section{Statistical Studies of SNRs}

Although some Galactic SNRs are identified at other than radio wavelengths, in practice the dominant selection effects are those that are applicable at radio wavelengths. Simplistically the two dominant selection effects are (e.g. Green 1991):

1. small SNRs less than a few arcmin in diameter are missing, as they are not resolved by surveys of the Galactic plane, and therefore they are not recognised as SNRs;

2. faint SNRs with surface brightnesses $\Sigma_{1 \mathrm{GHz}} \lesssim 8 \times 10^{-21} \mathrm{~W} \mathrm{~m}^{-2} \mathrm{~Hz}^{-1} \mathrm{sr}^{-1}$ are missing, as even if they are resolved by surveys, their intrinsic faintness makes it difficult to recognise faint SNRs in the presence of the confusing Galactic background.

Fig.1 shows histograms of the observed angular sizes and surface brightnesses for known Galactic SNRs in the 1998 version of my catalogue of Galactic SNRs (see "http://www.mrao.cam.ac.uk/surveys/snrs/"). 

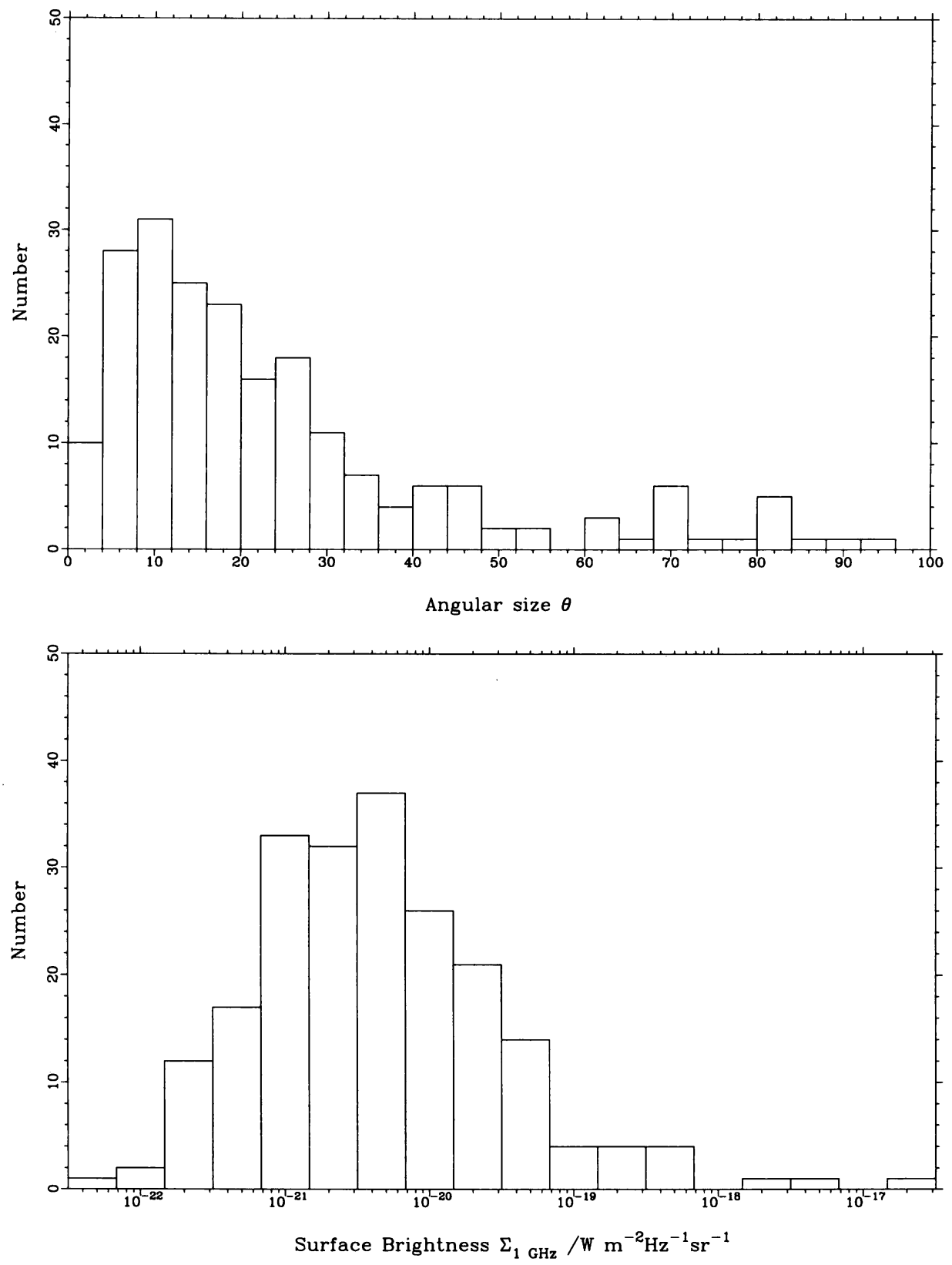

Figure 1. Histograms of (top) angular size and (bottom) surface brightness of known Galactic SNRs. 


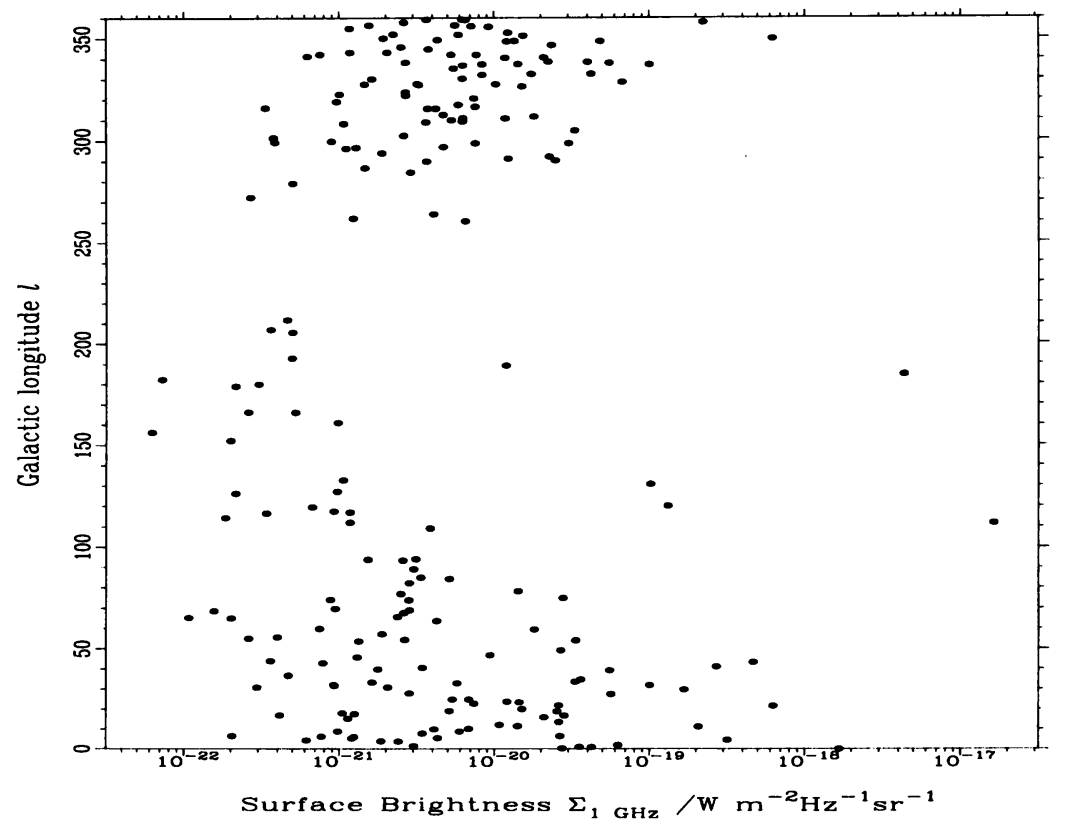

Figure 2. Plot of longitude against surface brightness of known Galactic SNRs.

The lack of small angular size remnants - i.e. young but distant remnants - is clear when the 'historical' remnants (Cas A, Tycho, Kepler, 3C58, the Crab Nebula, and the remnant of SN1006) are considered. These remnants are relatively close-by - as is expected, since their parent $\mathrm{SNe}$ were seen historically and therefore represent only a small fraction of the Galactic disk. Consequently we expect many more similar, but more distant remnants in our Galaxy (e.g. Green 1985), but these are not present in current catalogues. However, it should be noted that there are unlikely to be other luminous remnants in the Galaxy like Cas A and the Crab nebula that have been missed. On the other hand, the remnant of the SN1006 is faint, and more distant remnants similar to this would be very difficult to detect. There have been several searches for missing young but distant remnants (e.g. Green \& Gull 1984; Helfand et al. 1984; Green 1985, 1989; Sramek et al. 1992), but with only limited success. Such missing young but distant remnants are expected to have angular sizes of a few arcmin or less, and will not have been resolved sufficiently by single-dish surveys to be recognised as SNRs. Searching for these remnants is not easy because there are very many candidate sources in single-dish surveys to choose from, and they are likely to be in complex regions of the Galactic plane. The use of additional observational indicators (e.g. radio spectral index, infra-red to radio ratios etc) has not proved efficient for improving such searches, nor have the recent MOST survey (Whiteoak \& Green 1996) and the NVSS (Condon et al. 1998), which cover the Galactic plane with higher resolution than single-dish surveys, which is somewhat surprising. (Note, however, that the NVSS does contain previously 
unrecognised SNRs, for example near $l=354^{\circ}, b=-2^{\circ}$.) The fact that such missing remnants are likely to be in complex regions of the plane may mean that confusion is a very significant problem. A more radical alternative is that young but distant SNRs in the bulk of the Galaxy are not similar to Tycho, Kepler and 3 C58.

The difficulty in identifying intrinsically faint (i.e. low surface-brightness) remnants is complicated by the fact that this selection effect correlates with the with Galactic background, i.e. with $|b|$ and $|l|$. Fig.2 shows a plot of surface brightness against longitude of known Galactic SNRs. This clearly shows that there are relatively more faint SNRs identified in the 2nd and 3rd quadrants, which is where the Galactic background is less complicated, and therefore fainter SNRs are easier to identify. The fact that this selection effect correlates with $l$ (and $b$ ), means that it is difficult to disentangle this selection effect from, for example, the true 3-D distribution of SNRs in the Galaxy (e.g. Green 1996). Other observations, e.g. radio polarisation, infra-red, or good low-frequency radio observation, where there is less confusion with thermal emission, are required to get around this limit, although it will always be difficult to push this limit much lower in complex regions of the Galactic plane.

Many statistical studies of Galactic SNRs are limited by the lack of distance measurements for most SNRs. The ' $\Sigma-D$ ' relation has long been used to derive distances for individual SNRs, based on the correlation that large SNRs are fainter than small remnants. However, it needs to be remembered (e.g. Green 1991) that there is a wide range of intrinsic radio properties for SNRs of the same diameter. Fig.3 shows the $\Sigma-D$ relation for Galactic SNRs, which clearly shows a wide range of diameters for a given surface brightness. It should further be noted that the full range of intrinsic properties of SNRs may be even wider than that shown in Fig.3, as the selection effects discussed above mean that it is difficult to identify small and faint SNRs. Also, the apparent correlation shown in Fig. 3 is largely a consequence of the fact that it is a plot of surface-brightness, $\Sigma$, against diameter, $D$. Since $\Sigma$ depends on the flux density $S$ and angular size $\theta$ as $\Sigma \propto S / \theta^{2}$, and luminosity $L$ varies as $L \propto S d^{2}$, where $d$ is distance, then $\Sigma \propto L /(\theta d)^{2}$ or $\propto L / D^{2}$, which accounts for much of the correlation shown in Fig.3.

\section{Integrated Radio Spectra of SNRs}

Here I use the convention that the spectral index, $\alpha$, is defined so that the observed radio flux density varies as $\nu^{-\alpha}$. The radio spectra of SNRs can be summarised by the generalisation that "SNRs have a spectral index of 0.5 , as expected from Fermi acceleration". In practice, however, things are not that simple, and detailed studies of the spectra of SNRs are clearly important to the study the underlying physics of the particle acceleration in SNRs.

First, the spectral index of 0.5 applies only to shell SNRs, whereas filledcentre SNRs have flatter spectra, indicative of a quite different particle acceleration process at work. Second, the 0.5 is an average value for shell SNRs. For various reasons the uncertainties in the derived spectral indices of SNRs are often large - e.g. older data are not directly comparable with modern data; there are difficulties separating the SNR from complex background emission; there 


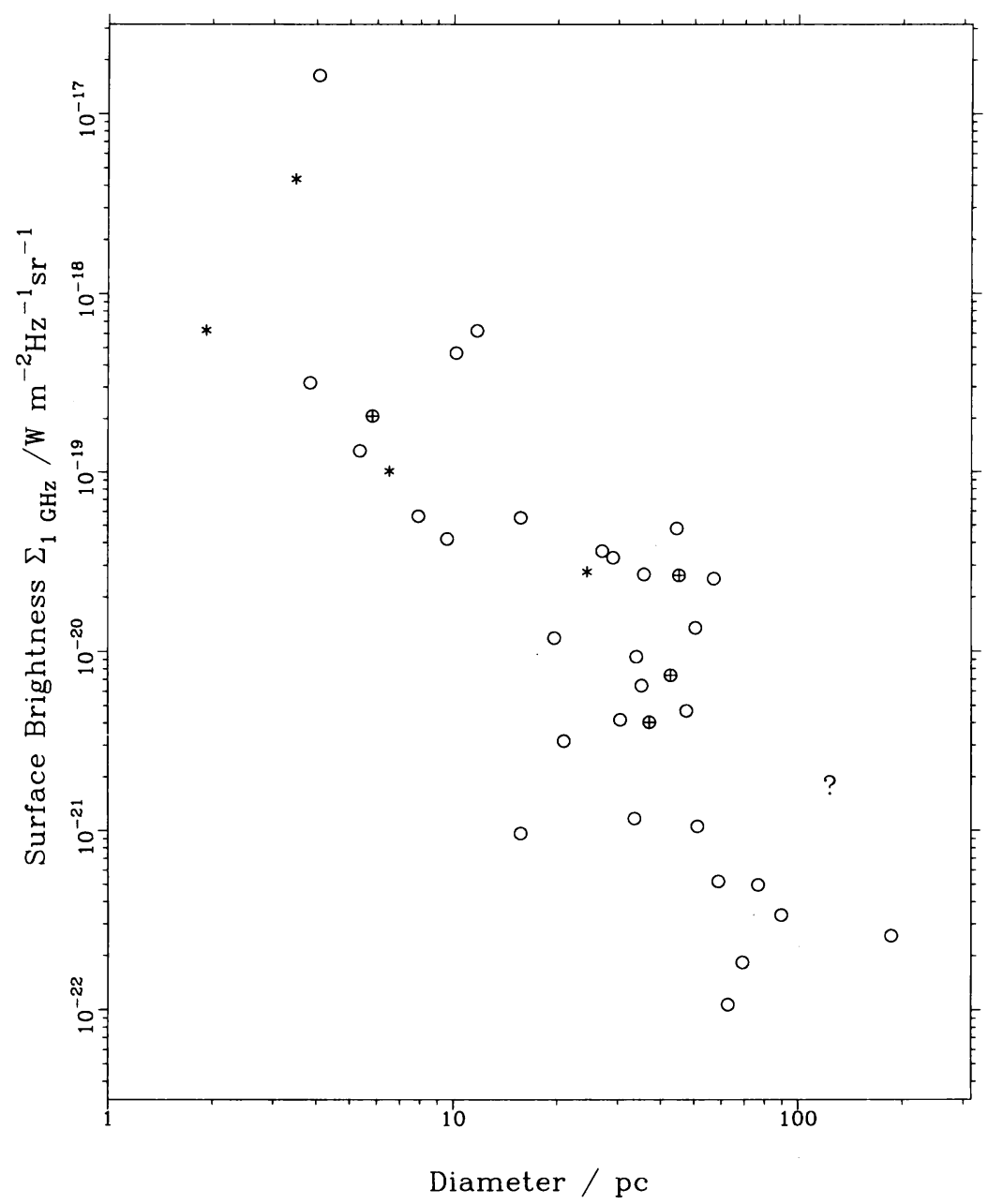

Figure 3. Plot of surface brightness against diameter for Galactic SNRs with known distances. 
are flux density scale uncertainties - but there are nevertheless shell SNRs with steeper and with flatter spectra than $\alpha=0.5$. Moreover, e.g. Green (1988), it is noticeable that known young shell remnants have spectra steeper than $\alpha \sim 0.5$. Third, the integrated spectra are not perfect power laws (e.g. low-frequency spectral turnovers due to thermal absorption, see Kassim 1989; high-frequency turnovers in filled-centre SNRs, see Morsi \& Reich 1987, Scheuer \& Green 1992). Finally, Cas A is fading, with a possible flattening of its spectrum with time, which complicates the flux-density scale of Baars et al. (1977) which is tied to measurements of Cas A. Some observations of Cas A (e.g. Rees 1990; Hook, Duffett-Smith, \& Shakeshaft 1992) imply that the flattening of the spectrum of Cas A is not as used by Baars et al. in their scale, and further observations are required both to understand how the radio emission from Cas $\mathrm{A}$ evolves, and also to better define flux density scales, especially at low frequencies.

For all these studies, the new, high-quality low-frequency observations from the GMRT will be useful, as many available low-frequency observations are of low resolution and were often made before the true extent of a remnant was well understood.

\section{Spectral Variations}

Spectral index variations across the faces of SNRs are expected for a variety of reasons, as seen from integrated spectral studies, namely: 1) flat-spectrum 'filled-centre' components of composite SNRs; 2) variations at high-frequencies in filled-centre remnants; 3 ) varying thermal absorption at low frequencies; and 4) differing shock conditions shell SNRs. Of these the last is potentially a very useful tool to study the underlying acceleration mechanism at work in SNR shocks. However, there is limited good evidence for variations across the face of remnants, because of observational difficulties. In general, good quality spectral index images require a wide range of frequencies, which in turn generally requires different telescopes and techniques, which introduce quite different systematic problems. (For example, interferometers do not measure large-scale structure, whereas single-dish 'basket-weaving' techniques filter large-scale structure from images).

Comparison, over small regions, using " $T-T$ " plots - i.e. plotting the brightness temperature at one frequency in a particular pixel against brightness temperature at another frequency for the same pixel - does help get around some of these systematic problems. Ideally, the gradient of the resulting straight line depends on spectral index, with base level offsets in images corresponding to changes in the intercepts. Thus, provided the regions chosen are not too large, so the base level offsets do not change much, and there is a large enough range of intensities for the emission from the remnant, then this method is useful for spectral index studies.

Given the limited space available here I will only briefly review the results for spectral index variations across some young, bright remnants, and for faint remnants in unconfused regions of the Galactic plane.

For young remnants, the most precise spectral index studies available have been of Cas A, and the Crab nebula. Detailed studies of VLA images of Cas A (Anderson et al. 1991; Anderson \& Rudnick 1996) have revealed steeper spectra 
knots at larger projected radii, and steeper spectra for the brighter knots. Anderson \& Rudnick conclude that the lack of correlation between spectral index and dynamical properties of knots means that the knots are not currently active sites of particle acceleration. In addition, Kassim et al. (1995), from VLA images at 74 and $333 \mathrm{MHz}$, detected spectral flattening near the centre of Cas $\mathrm{A}$, probably due to absorption by ionised gas in the remnant. For the Crab Nebula, much effort has been expended on investigating whether or not the spectral index changes near the edge of the remnant, as studies of this would provide a valuable constraint on the acceleration and diffusion of particles in the nebula. However, it is exactly this sort of variation which can result from any systematic differences in the details of how images are produced at different frequencies. Indeed, although such variations have been reported, they are not supported by the most recent observations by Bietenholz et al. (1996). However, Bietenholz et al. did detect spectral variations at low frequencies due to absorption by the thermal filaments in the Crab nebula, and variations near the central pulsar, possibly associated with the 'wisp'.

For older, fainter remnants, it is best to study remnants that are away from complex regions in the Galactic plane. Furthermore, in order to understand any detected variations in terms of differences in the shock conditions in different regions of the remnants it is best to select remnants that are asymmetric. Two remnants which match these conditions, and for which detailed spectral index studies have been made are IC443 (=G189.1+3.0) and the Cygnus Loop (=G74.0-8.5). IC443 has a brighter, smaller NE rim, which shows a possibly steeper spectrum than the fainter, larger SW rim (Green 1986). However, more striking in Green's results is the flat spectrum region in the south, which has recently been associated with a hard-spectrum X-ray emission by Keohane et al. (1997). This is also the region interacting with surrounding molecular material, which seems indicative of a site of ongoing acceleration. For the Cygnus Loop, $T-T$ plots - see Leahy \& Roger (1998) and also Green (1990) - show both spectral variations and evidence for spectral breaks in the radio emission.

\section{Conclusions}

For the identification of Galactic SNRs, and therefore for the statistical studies of them, selection effects are important. Both intrinsically low-surface brightness and young but distant (i.e. small) high-surface brightness remnants are missing from current catalogues of Galactic SNRs. Further high-resolution observations of sources in the Galactic plane are required to identify the missing young remnants in the Galactic plane. This is important to understand the range of intrinsic properties of SNRs, which limits many statistical studies. Less easy is the identification of fainter remnants - a wide range of observations, including better low-frequency radio observations will, however, help disentangle faint SNRs from confusing emission. Additionally, better low-frequency observations of Galactic SNRs, as will no doubt be provided by GMRT observations in the coming years, will also help both to define better their integrated spectra, and to study spectral variations better. 
Acknowledgments. It is a pleasure to thank many colleagues, both at MRAO and elsewhere, for many interesting conversations on diverse topics related to Galactic SNRs.

\section{References}

Anderson, M.C. \& Rudnick, L. 1996, ApJ, 456, 234

Anderson, M. Rudnick, L. Leppik, P. Perley, R. \& Braun, R. 1991, ApJ, 373, 146

Baars, J.W.M. Genzel, R. Pauliny-Toth, I.I.K. \& Witzel, A. 1977, A\&A, 61, 99

Bietenholz, M.F. Kassim, N. Frail, D.A. \& Perley, R.A. Erickson, W.C. Hajian, A.R. 1997, ApJ, 490, 291

Condon, J.J., Cotton, W.D., Greisen, E.W., Yin, Q.F., Perley, R.A., Taylor, G.B. \& Broderick, J.J. 1998, AJ, 115, 1693

Green, D.A. 1985, MNRAS, 216, 691

Green, D.A. 1986, MNRAS, 221, 473

Green, D.A. 1988, in NATO ASI C220, Genesis and Propogation of Cosmic Rays, ed. M.M. Shapiro \& J.P. Wefel (Reidel: Dordrecht), 205

Green, D.A. 1989, AJ, 98, 1358

Green, D.A. 1990, AJ, 100, 1927

Green, D.A. 1991, PASP, 103, 209

Green, D.A. 1996, in IAU Coll. 145, Supernovae and Supernova Remnants, ed. R. McCray \& Z. Wang (CUP: Cambridge), 341

Green, D.A. \& Gull, S.F. 1984, Nature, 312, 527

Green, D.A. \& Scheuer, P.A.G. 1992, MNRAS, 258, 833

Helfand, D.J. Chance, D. Becker, R.H. \& White, R.L. 1984, AJ, 89, 819

Hook, I.M. Duffett-Smith, P.J. \& Shakeshaft, J.R. 1992, A\&A, 255, 285

Kassim, N.E. 1989, ApJ, 347, 915

Kassim, N.E. Perley, R.A. Dwarakanath, K.S. \& Erickson, W.C. 1995, ApJ, 455, L59

Keohane, J.W. Petre, R. Gotthelf, E.V. Ozaki, M. \& Koyama, K. 1997, ApJ, 484,350

Leahy, D.A. \& Roger, R.S. 1998, ApJ, 505, 784

Morsi, H.W. \& Reich, W. 1987, A\&AS, 69, 533

Rees, N. 1990, MNRAS, 243, 637

Sramek R.A. Cowan, J.J. Roberts, D.A. Goss, W.M. \& Ekers, R.D. 1992, AJ, 104, 704

Whiteoak, J.B.Z. \& Green, A.J. 1996, A\&AS, 118, 329 